\title{
Specific Morphology of Polyethylene Blown Films Discovered Using a Convergent Beam Optical Microscope
}

\author{
S. Utsumi,* M. Minobe, ${ }^{* *}$ and N. Yamaguchi* \\ *Sumitomo Chemical Co., Ltd., Petrochemicals Research Laboratory, 2-1 Kitasode Sodegaura city, \\ Chiba 299-0295, Japan \\ **Sumitomo Chemical Co., Ltd., Head Office (Tokyo), Tokyo Sumitomo Twin Building (East), 27- \\ 1, Shinkawa 2-chome, Chuo-ku, Tokyo 104-8260, Japan
}

1. Introduction For analyzing morphologies of various materials with certain regularity, we developed a new microscope, which we call a convergent beam optical microscope (CBOM) [1]. We analyzed morphologies of blown films of linear low-density polyethylene (LLDPE) / low-density polyethylene (LDPE) blends, and studied effect of morphologies on transparency of films with CBOM. Haze of blown films depends on LDPE content, and its minimum value is given in a particular LDPE content $(10-20 \mathrm{wt} \%)$, that is, the films are the most transparent in that content. It was indicated by the study with a scanning electron microscope (SEM) that the increase of haze in lower content was mainly due to the formation of spherulites, but SEM did not clarify the cause of the increase of haze in higher content. Therefore, we analyzed these phenomena using CBOM.

2. Convergent beam optical microscope (CBOM) Conventional optical microscopes normally use nearly parallel beam as illumination light, but CBOM uses convergent beam, which enables one to carry out several novel observations as below: (1) CBOM makes it possible to obtain both diffraction (or scattering) patterns and real space images, (2) By setting a suitable spatial filter on the diffraction pattern, we can obtain the corresponding real space image formed by the light passed through it.

3. Experiments CBOM was manufactured by Nikon engineering Co., Ltd., and equipped with some special parts as blow: (1) a point light source, which consisted of a $100 \mathrm{~W}$ halogen lump and a plate with a $100 \mathrm{~mm} \phi$ pinhole in the center, (2) a condenser lens converging incident light from the point light source, and (3) a spatial filter, which was a plate with a $800 \mu \mathrm{m} \phi$ pinhole in the center. Blown films of LLDPE / LDPE blends with LDPE content of 0, 10, 50, and $100 \mathrm{wt} \%$ were observed.

4. Results and discussion It is well known that haze of films increases according as their own scattering. Because CBOM made it possible to directly correlate light scattering patterns with their corresponding real space images, we tried to analyze the morphology of blown films of LLDPE / LDPE blends in higher LDPE content with CBOM. The films of blends in more than $50 \mathrm{wt} \% \mathrm{LDPE}$ content showed characteristic scattering patterns, and were found to form a 'net-like structure' in their corresponding real space images given by scattered light in meridian direction with CBOM (Fig.), whereas the structure could not be observed with a conventional optical microscope. This morphology was considered to be one of the main factors to cause light scattering and to increase the haze, because the scattering patterns were spread more widely according to magnitude of haze. The 'net-like structure' was considered to exist in surface morphology of the films, since this morphology was eliminated when the films were enclosed in dimethyl phtharate, which had diffractive index nearly equal to that of the films. Furthermore, we confirmed the existence of the 'net-like structure' in surface morphology of the films with an atomic force microscope, which is based on a quite different principle from CBOM. The 'net-like structure' on polyethylene blown films seems to be found for the first time. Another morphology, 'vertical stripes structure', was observed with CBOM in the surface morphology of the films, but it was not considered to increase the haze of the films.

\section{References}

[1] S. Utsumi, et al., the $14^{\text {th }}$ International Symposium on Polymer Analysis and characterization, Nagoya, June 6-8, 2001, p.91. 

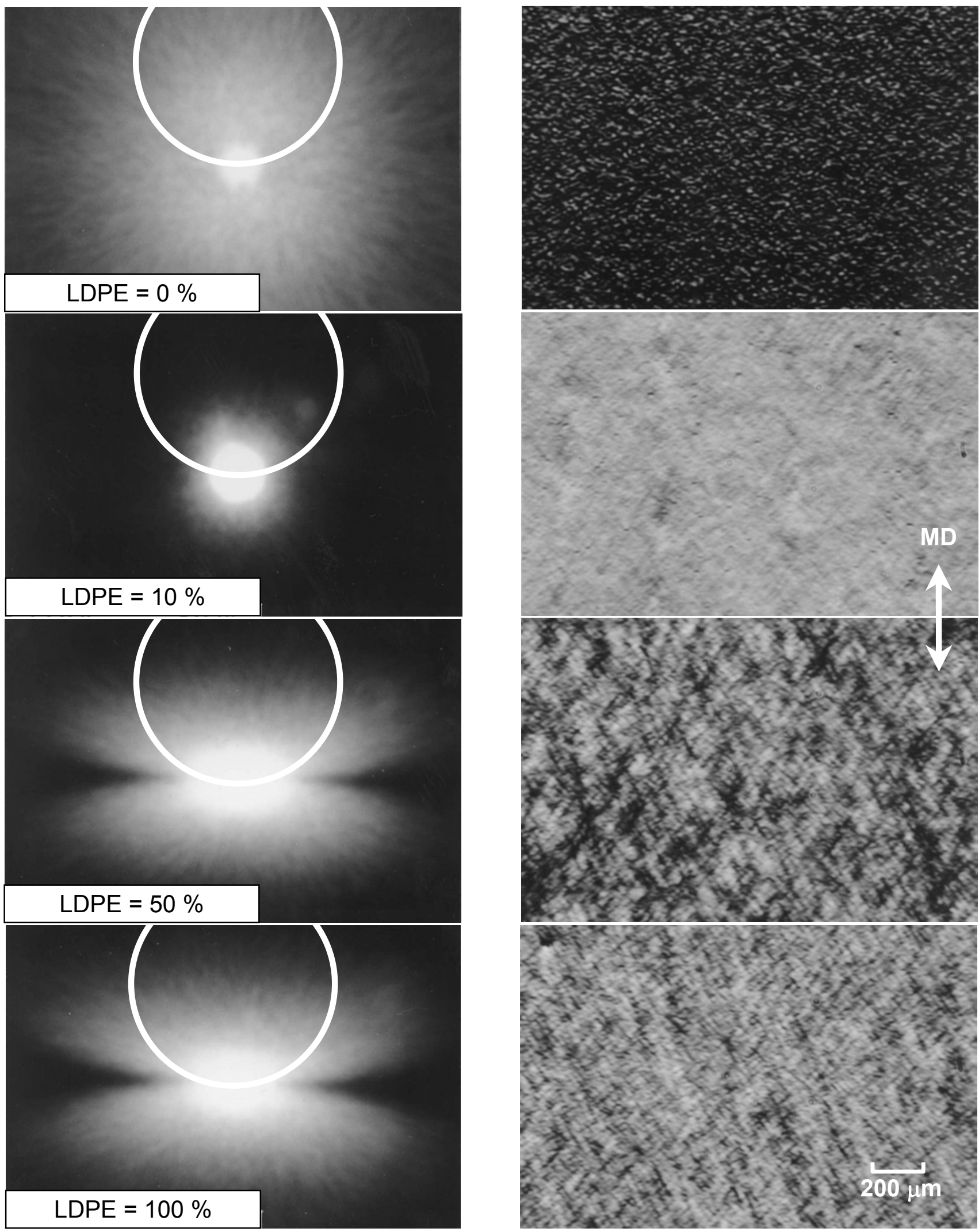

Fig. CBOM images of blown films of LLDPE / LDPE blends. (Left) Light scattering patterns. (Right) Real space images formed by light within circles in the left images. (MD: Machine direction) 